\title{
Review of: "Hemorrhagic stroke treated by transcranial neuroendoscopic approach"
}

\author{
Cheng-Di Chiu
}

Potential competing interests: The author(s) declared that no potential competing interests exist.

1. In this article, authors demonstrated several causes of ICH dealing with endoscopic or microscopic surgery. The surgical pictures are beautiful and authors announced encouraging results. However, hemorrhagic stroke treat by endoscope is not a new technique, and several large series reports, real world data and clinical trials already been published which made this manuscript is short of novity.

2. Besides, based on the authors provided results, their technique is just not inferior to others, no known outstanding part that can be traced.

3. Authors made a good summary of their surgical experiences in either endoscope or microscope or both in several etiologies of cerebral hemorrhage. However, individual detail information is lacking and scientific evidence is inadequate.

4. In the conclusion paragraph, authors declared that "In PHS, transcranial neuroendoscopy was a superior technique in cases of subcortical, intraventricular, and cerebellar hemorrhage and a good technique in cases of basal ganglia hemorrhage. In SHS, transcranial neuroendoscopy was superior in cases of $\mathrm{CH}$ and aneurysms and good in cases of AVMs/AVFs. However, some aneurysms and most AVMs/AVFs require ECM.", but I did not see any comparative result lead to this conclusion.

As above reasons, I did not think this article is qualified to be published in this high quality journal. 